\title{
Neonatal bacterial meningitis: Results from a cross-sectional hospital based study
}

\author{
Izeta Softić' ${ }^{*}$, Husref Tahirović ${ }^{2}$, Mensuda Hasanhodžić ${ }^{1}$
}

${ }^{1}$ Department of Paediatrics University Clinical Centre Tuzla, Tuzla, Bosnia and Herzegovina, ${ }^{2}$ Department of Medical Sciences, Academy of Sciences and Arts of Bosnia and Herzegovina, Sarajevo Bosnia and Herzegovina

\footnotetext{
${ }^{\star}$ Corresponding author: izeta.softic@bih.net.ba Tel.: + 38735303714

Fax.: + 38735303740
}

Received: 6 May 2015

Accepted: 16 November 2015

Key words: Meningitis - Newborn Neonatal intensive care unit.

\begin{abstract}
Objective. The aim of the study was to determine the epidemiological characteristics of bacterial meningitis observed in neonates born in the Department of Gynaecology and Obstetrics, University Clinical Centre Tuzla, Bosnia and Herzegovina, admitted to Intensive care unit (NICU) or readmitted, because of suspected infection, after discharge from the nursery. Subjects and methods. This study was carried out from July 1, 2012 to June 30, 2013. During this period 4136 neonates were born. All neonates admitted to the Intensive care unit with signs and symptoms of systemic infections, and neonates readmitted to the Intensive care unit, after discharge from the nursery for sepsis work up were included in the study. Results. Eighteen of 200 neonates (9\%) admitted or readmitted to the NICU developed meningitis. $61 \%$ cases were late onset meningitis. The overall incidence was 4.4/1000 live births. The mortality rate was $11.1 \%$. The mean age of symptom presentation was 8.7 days. The most common clinical features were: fever, respiratory distress and jaundice. Significant risk factors for acquiring meningitis were: male gender, Caesarean delivery, stained amniotic fluid. Positive CSF finding were detected in 6/18 (33.3\%) of cases. Gram-positive bacteria were more frequently responsible for confirmed meningitis. In all neonates with meningitis blood culture was examined and 5 (50\%) yielded Gram-negative bacteria. Conclusion. The high rates of neonatal meningitis with predominant late onset may suggest nosocomial origin. Measures to improve antenatal, intrapartum and delivery care and measures during NICU hospitalisation are necessary to lower the risk of nosocomial infections.
\end{abstract}

\section{Introduction}

Acute bacterial meningitis is more frequent in the neonatal period than in any other time of life and leads to a high incidence of mortality and long term neurological sequels. The incidence of neonatal meningitis is variously calculated at between 0.25 and 0.32 per 1000 live births, depending on the inclusion criteria (1). In resource - poor countries the incidence of neonatal bacterial meningitis may be underestimated (2).
In neonates with documented sepsis and in preterm infants the incidence is significantly higher. Neonates are at higher risk of meningitis because of immaturity of humoral and cellular immunity, and the absence of specific clinical signs makes diagnosis of meningitis more difficult in neonates than in older children (3). A recent review of the incidence of neonatal meningitis infections reported 0.8 to 6.1 cases every 1,000 live birth (4). Observational studies have shown that in developing countries Klebsiella pneu- 
moniae, Serratia species and other Gramnegative bacteria are to date the leading bacterial agents of neonatal meningitis, while in Europe, America and Australia, Group B Streptococcus is predominant (5). There is no data on the incidence of neonatal meningitis in Bosnia and Herzegovina.

The aim of the study was to determine the incidence, aetiology, risk factors and outcome of bacterial meningitis in neonates born in Department of Gynaecology and Obstetrics, University Clinical Centre Tuzla, who were admitted to the Intensive care unit of the Department of Paediatrics for suspected infection.

\section{Subjects and methods}

This cross-sectional study involved neonates born in Department of Gynaecology and Obstetrics, University Clinical Centre, Tuzla, over a one-year period, from July 1, 2012 to June 30,2013 , who were admitted to the Intensive care unit for sepsis work up. Neonates were also included in the study after discharge from nursery and who were readmitted to the Intensive care unit with signs and symptoms of systemic infection.

\section{Context of the study}

The Department of Obstetrics and Gynecology has 4500 deliveries annually. The mothers and their babies stay in hospital for one day in normal newborn nurseries. Rooming-in was introduced in 2004, and breastfeeding is supported for well babies, but there is no feeding with fresh human milk for sick babies in the Intensive care unit.

The Neonatal Intensive Care Unit (NICU) of Department of Paediatrics provides intensive care for 18 patients. It is organized as follows: it has an average of 10 nurses, backed up by a neonatologist on a daily basis, with four intensive care physicians working in the NICU, and one on duty 24-hours. The NICU has an area of $293 \mathrm{~m}^{2}$, it has one common and two isolation rooms, with two shared sinks and one in each isolation room. The admittance of mothers and visitors is limited to once per day for visiting and information about the baby. There is a filter before entering the ward area, equipped with a hand washing station, where all visitors are required to wash their hands. For almost all newborn, antibiotics are routinely used before entering the NICU.

\section{Patients}

In the study we enrolled 200 neonates born in the Obstetric Unit and admitted to the Intensive care unit due to various clinical problems. We also included neonates discharged from the nursery and readmitted to the Intensive care unit for sepsis work up. For each enrolled neonate with meningitis, controls were selected by the random selection method from neonates admitted to the Intensive care unit, who were without infection. Two controls were used per study case, giving a total of 36 controls.

We recorded demographic characteristics, birth weight, gestational age, gender, Apgar score, and mode of delivery. We also recorded maternal risk factors, as well as maternal hospitalisation during pregnancy, fever, and premature rupture of membranes (PROM), modality of delivery, and the presence of stained amniotic fluid.

For neonates classified as having meningitis we recorded the date of onset of the infection, symptoms at the onset of infection, microbiological data of cerebrospinal fluid (CSF), blood cultures and CSF index. Neonates were defined as having meningitis if they presented clinical symptoms of sepsis and the cerebrospinal fluid (CSF) analysis showed one or more of the following laboratory signs: 1) positive microbiological cultures 2) CSF index suggestive of bacterial process, as reported by Sarff et al. (6) con- 
sidering the GA, and by Rodriguez et al. (7) considering the post-conceptional age.

Presentation during the first week of life was defined as early onset meningitis (EOM), while late onset meningitis (LOM) included presentation between the $8^{\text {th }}$ and $30^{\text {th }}$ postnatal days. Infection was considered nosocomial if it was diagnosed after 48 hours of maternal hospitalisation and subsequent birth, or after 48 hours of hospitalisation of the newborn (8). Blood culture was obtained for each neonate with signs of sepsis by the method described by Buttery (9). The blood specimen was further inoculated into a BACTEC Peds plus/F culture vial (BACTEC, Becton Dickinson, USA) and inoculated cultures were incubated as soon as possible in a BACTEC 9120 instrument for up to five days, as recommended by Becton Dickinson Microbiological Systems (10). PROM was defined as rupture of the membranes prior to 18 hours before delivery (11). The mortality rate was also calculated.

\section{Ethics statement}

The study was approved by the Ethics Board of University Clinical Centre Tuzla, Tuzla, Bosnia and Herzegovina. All procedures were in accordance with the Declaration of Helsinki. Informed consent was not required because all information was de-identified.

\section{Statistical analyses}

The results were analysed by arithmetic mean and standard deviation. Inferences about categorical data were analyzed using the chi-square. The association of risk factors with the development of meningitis is the frequency of each characteristic among meningitis group divided by the frequency of the same characteristic in the group of controls with no infection. This is the relative risk of infection associated with each variable. Analyses were performed by IBM
SPSS Statistics 22 and MedCalc statistical software for Windows Version 13-14.10.2. $P$ values were considered statistically significant at a value of 0.05 .

\section{Results}

From July 1, 2012 to June 30, 20134136 neonates were born and 200 were hospitalised in the NICU. Diagnosis of meningitis was performed in 18 infants. The incidence of meningitis was 4.4/1000 live birth. The number of neonates hospitalised in the NICU with meningitis was nine, and after discharge from the nursery nine neonates were readmitted for meningitis. Risk factors for acquiring meningitis in neonates are shown in Table 1.

Birth weight (BW) and gestational age (GA) were similar between neonates with meningitis and in neonates of control group: $2867 \pm 835$ g (range: $1100-4100$ g) v. $2919 \pm 850$ (range, 1300-4500); and 36.3 weeks (range: 30-40 weeks) v. 37.0 (range, 30-40 weeks). Half the neonates were preterm but only two showed BW $\leq 1500$ g (11.1\%). A significantly greater number of neonates with Caesarean section and with stained amniotic fluid was present in cases in comparison to the controls $(\mathrm{p}<0.05)$, as well as a greater number of male neonates $(\mathrm{p}<0.001)$.

Mean age at presentation was 8.7 days of life (range: 1-28 days). Seven of 18 the neonates with meningitis (38.9\%) had EOM and 11 LOM (61.1\%). Admission of neonates $\geq 7$ days was significantly more frequent in the group of neonates with meningitis compared to those without meningitis $(\mathrm{p}<0.001)$.

The most common clinical features were fever $(27.8 \%)$, respiratory distress $(22.2 \%)$ and jaundice (16.7\%), while other clinical findings of meningitis, such as apnoea, convulsions and irritability were less common (Table 2).

The mean leukocyte count in CSF was $498 \pm 1178$ (5-5000). CSF leukocyte count was more than $1000 / \mathrm{mm}^{3}$ in $3(16.6 \%)$ neo- 
Table 1 Risk factors for acquiring neonatal meningitis

\begin{tabular}{|c|c|c|c|c|c|}
\hline \multirow{3}{*}{ Risk factors } & \multicolumn{2}{|c|}{ Population of neonates } & \multirow{3}{*}{$\mathrm{RR}$} & \multirow{3}{*}{$95 \% \mathrm{Cl}$} & \multirow{3}{*}{$\mathrm{p}$} \\
\hline & Cases $(n=18)$ & Controls $(n=36)$ & & & \\
\hline & n (\%) & $\mathrm{n}(\%)$ & & & \\
\hline \multicolumn{6}{|l|}{ Infants } \\
\hline Sex (male) & $15(83.3)$ & $9(25)$ & 3.3 & $1.825-6.088$ & $<0.001$ \\
\hline BW $<1501 \mathrm{~g}$ & $2(11.1)$ & $1(2.8)$ & 4 & $0.388-41.23$ & $>0.05$ \\
\hline $\mathrm{GA} \leq 32$ weeks & $2(11.1)$ & $1(2.8)$ & 4 & $0.388-41.23$ & $>0.05$ \\
\hline AS at $1 \min \leq 5$ & $3(16,7)$ & $5(13.9)$ & 1.2 & $0.322-4.469$ & $>0.05$ \\
\hline$A A \geq 7$ days & $11(61.1)$ & $1(2,8)$ & 22 & $3.076-157.34$ & $<0.001$ \\
\hline \multicolumn{6}{|l|}{ Mothers } \\
\hline Caesarean delivery & $8(44.4)$ & $5(13.9)$ & 3.2 & $1.221-8.387$ & $<0.05$ \\
\hline Stained amniotic fluid & $6(33.3)$ & $3(8.3)$ & 4 & $1.129-14.175$ & $<0.05$ \\
\hline PROM & $4(22.2)$ & $3(8.3)$ & 2.6 & $0.667-10.663$ & $>0.05$ \\
\hline$H \geq 24$ h before delivery & $2(11.1)$ & $1(2.8)$ & 4 & $0.388-41.23$ & $>0.05$ \\
\hline
\end{tabular}

$\mathrm{BW}=$ Birth weight; $\mathrm{GA}=\mathrm{Gestational}$ age; $\mathrm{AS}=$ Apgar score; $\mathrm{AA}=$ Age of admission; $\mathrm{H}=$ Hospitalisation; $\mathrm{PROM}=$ Premature rupture of membrane; $\mathrm{RR}=$ Relative Risk; $\mathrm{Cl}=95 \%$ confidence intervals.

Table 2 Clinical manifestations in newborn with meningitis

\begin{tabular}{ll}
\hline Manifestations & $\mathrm{n}(\%)$ \\
\hline Respiratory distress & $4(22.2)$ \\
Apnoea & $2(11.1)$ \\
Fever & $5(27.8)$ \\
Irritability & $2(11.1)$ \\
Convulsions & $2(11.1)$ \\
Jaundice & $3(16.7)$ \\
\hline Total & $18(100)$ \\
\hline
\end{tabular}

nates, and more then $100 / \mathrm{mm}^{3}$ in $8(44.4 \%)$. Two neonates had leukocyte count within the reference range, but both had positive CSF culture and clinical manifestations of infection. The mean glucose levels, CSF glucose/blood glucose ratio and protein levels were $33 \pm 12(5-56) \mathrm{mg} / \mathrm{dl}, 0.45 \pm 0.21$ $(0.7-0.77)$ and $153 \pm 65(87-313) \mathrm{mg} / \mathrm{dl}$. The bacterial isolates from CSF and blood in the neonates are shown in Table 3.

Bacterial CSF culture was positive in 6 neonates (33.3), showing growth of Gram-positive bacteria in 5 neonates $(27.7 \%)$ and Gramnegative bacteria in 1 (5.5\%). Blood culture was examined in all neonates; 10 of them had
Table 3 Bacteria isolated from CSF and blood in 18 neonates with meningitis

\begin{tabular}{lll}
\hline Isolates bacteria & CSF (n) & Blood (n) \\
\hline Listeria monocytogenes & 1 & - \\
Acinetobacter baumannii & - & 4 \\
Enterococcus faecalis & 1 & 2 \\
Enterobacter cloacae & 1 & 1 \\
Streptococcus species & 2 & - \\
Coagulase-negative & 1 & 3 \\
Staphylococcus & & \\
\hline
\end{tabular}

CSF=Cerebrospinal fluid.

positive culture; Gram-positive bacteria were isolated from 5 (27.7\%) and Gram-negative bacteria from 5 (27.7\%). In two neonates, no bacteria was isolated from the CSF or blood, but pleocytosis and low glucose were found in one, and pleocytosis and high protein level in the other. The mortality rate was $11.1 \%$ in neonates with meningitis, and $4.6 \%$ in the group without infection.

\section{Discussion}

This is the first epidemiological study of neonatal meningitis in Bosnia and Herzegovina. The incidence was $4.4 / 1000$. This 
high incidence of neonatal meningitis is in keeping with the high incidence of neonatal sepsis (32.8/1000 live births, unpublished data) and with the incidence in developing countries $(12,13,14)$. There is no feeding with fresh human milk for sick babies in our Intensive care unit, but it does exist in the NICU where $98 \%$ of mothers provide milk for their infants (15). This is a preventive measure to reduce the risk of infections (16). In almost all newborn, antibiotics are routinely used before entering the NICU, increasing the risk of opportunistic infection and development of antibiotic resistant organisms over time (17). Supporting breastfeeding in the NICU and antibiotic stewardship are effective clinical strategies to reduce the high incidence of neonatal sepsis and meningitis in our neonatal units.

Our data indicated the predominance of the male gender, with similar findings to Aletayeb et al. (18). The greater susceptibility of males is evident in cases of sepsis and meningitis acquired during delivery or in nurseries (19). Late onset meningitis was predominant. This finding is in keeping with the high incidence of late onset neonatal sepsis in our investigation (48.5\%, unpublished data). Confirmed meningitis caused by Listeria monocytogenes in our study had a nosocomial origin, because in the same period a neonate was born with confirmed Listeria monocytogenes sepsis from a twin pregnancy and complicated Caesarean delivery. The first twin had Listeria monocytogenes sepsis, but the second was healthy. The neonate with Listeria monocytogenes meningitis had a Bruton agammaglobulinemia like predisposing risk for acquiring infection. Late onset and cross-infection of Listeria monocytogenes meningitis was confirmed from other authors $(20,21)$. Four neonates with meningitis had confirmed sepsis with Acinetobacter baumannii. In the same period we documented an outbreak of Acinetobacter baumannii in the Intensive care unit (22). The Gram-negative bacteria (Enterobacter cloacae), isolated from liquor and blood, Acinetobacter baumannii isolated from blood, and the more frequent late onset meningitis, indicate a nosocomial origin, similar to the investigation by Aletayeb et al. (18).

Caesarean delivery and stained amniotic fluid were significant risk factors for acquiring meningitis. Caesarean section is associated with more neonatal sepsis compared to normal vaginal delivery (23). In our study, stained amniotic fluid was significantly frequent in neonates with meningitis. According to the investigation by Zanella et al. (24), stained amniotic fluid was one of the obstetric risk factors for chorioamnionitis, together with frequent digital vaginal examination, and the duration of labour, with the increased risk of neonatal sepsis and meningitis. Obstetric risk factors are very important for distinguishing the origin of neonatal infection. According to the Centre for Disease Control definition, newborn infection that is the result of passage through the birth canal is considered nosocomial, and no specific time during or after hospitalisation is given to determine whether an infection is nosocomial (8). The limitation of our results according to the risk factors for acquiring meningitis might be the matching of controls with a different sex, the type of delivery or day of admission.

Fever was the most presented symptom. The late onset of meningitis and low rate of VLBW infants in our study group may be an explanation. Fever was the most common clinical feature in term-delivered neonates with meningitis in the investigation by Kavuncuoğlu et al. (13). The mortality rate was $11.1 \%$. Compared with developed countries, it is similar (2), but the period of investigation was only one year, and there are no previous data for comparison.

In summary, in a one-year investigation, from July 1, 2012 to June 30, 2013, 4.4/1000 
live newborn were diagnosed as having meningitis. The incidence of meningitis was high compared with the incidence in the developed world. The late onset of symptoms, risk factors and type of isolated bacteria may indicate a nosocomial origin. The mortality rate was $11.1 \%$. Measures to improve antenatal, intrapartum and delivery care, and measures during NICU hospitalisation are necessary to lower the risk of nosocomial infections.

\section{Conclusions}

Important differences have been identified in the incidence, aetiology and risk factors in our study, compared with the results in developed countries. Measures to improve antenatal, intrapartum and delivery care, and measures during NICU hospitalisation are necessary to lower the risk of nosocomial infections.

\section{What is already known on this topic \\ Bacterial meningitis is still a major cause of morbidity in neo- nates in the developing world. Differences in the epidemiology of neonatal bacterial meningitis have been identified between developed and developing countries. In our region no epidemi- ological study has been conducted of neonatal bacterial men- ingitis.}

\section{What this study adds}

The results of our study do not add to general knowledge regarding the of epidemiological characteristics of neonatal bacterial meningitis, but they are important as a very careful analysis and present the epidemiology of neonatal bacterial meningitis and risk factors in this region.

Authors' contributions: Conception and design: IS, HT; Acquisition, analysis and interpretation of data: IS, MH; Drafting the article: IS, HT; Revising it critically for important intellectual content: IS, HT.

Conflict of interest: The authors declare that they have no conflict of interest.

\section{Reference}

1. De Louvois J. Acute bacterial meningitis in the newborn. J Antimicrob Chemother. 1994;34:6173.
2. Faryk JS, Swan O, Molineux E. Sytematic review: neonatal meningitis in the developing world. Trop Med Int Health. 2011;16:672-9.

3. Baud O, Aujard Y. Neonatal bacterial meningitis. Handb Clin Neurol. 2013;112:1109-13.

4. Thaver D, Zaidi AK. Burden of neonatal infections in developing countries: a review of evidence from community-based studies. Pediatr Infect Dis J. 2009;28:3-9.

5. Al-Harthi AA, Daqriri KA, Asindi AA, Bello CS. Neonatal meningitis. Neurosciences (Riyadh). 2000;5:162-5.

6. Sarff LD, Platt LH, McCracken GH Jr. Cerebrospinal fluid evaluation in neonates: comparison of high-risk infants with and without meningitis. J Pediatr. 1976;88(3):473-7.

7. Rodriguez AF, Kaplan SL, Mason EO Jr. Cerebrospinal fluid values in the very low birth weight infant. J Pediatr. 1990;116(6):971-4.

8. Garner JS, Jarvis WR, Emori TG, Horan TC, Hughes JM. CDC definitions for nosocomial infections, 1988. Am J Infect Control. 1988;16(3):12840.

9. Buttery JP. Blood cultures in newborns and children: Optimizing an everyday test. Arch Dis Child Fetal Neonatal Ed. 2002;87:F25-8.

10. Becton Dickinson Microbiological Systems. Bectec PEDS PLUS/F culture vials: Instruction leaflet. Sparks, Mariland: Becton Dickinson Company; 2000.

11. Herbst A, Källén K. Time between membrane rupture and delivery and septicemia in term neonates. Obstet Gynecol. 2007;110(3):612-8.

12. Ali Z. Neonatal meningitis: a 3-year retrospective study at the Mount Hope Women's hospital, Trinidad, West Indies. Journal Journal of Tropical Paediatric. 1995;41:109-11.

13. Kavuncuoğlu S, Gürsoy S, Türel Ö, Aldemir EY, Hoşaf E. Neonatal bacterial meningitis in Turkey: epidemiology, risk factors, and prognosis. J Infect Dev Ctries. 2013;15:7(2):73-81.

14. Khalessi N, Afsharkhas L. Neonatal meningitis: risk factors, causes, and neurologic complications. Iran J Child Neurol. 2014;8(4):46-50.

15. Meier PP, Patel AL, Bigger HR, Rossman B, Engstrom JL. Supporting breastfeeding in the neonatal intensive care unit: Rush Mother's Milk Club as a case study of evidence-based care. Pediatr Clin North Am. 2013;60(1):209-26.

16. Manzoni P, De Luca D, Stronati M, Jacqz-Aigrain E, Ruffinazzi G, Luparia M, et al. Prevention of nosocomial infections in neonatal intensive care units. Am J Perinatol. 2013;30(2):81-8. 
17. Stewart L, Skeoch CH, Jones B. Rationing of antibiotic use in neonatal units. Arch Dis Child Fetal Neonatal Ed. 2001;84(3):F218

18. Aletayeb MH, Ahmad FS, Masood D. Eleven-year study of causes of neonatal bacterial meningitis in Ahvaz, Iran. Pediatr Int. 2010; 52 (3):463-6.

19. Klein JO, Marcy SM. Bacterial Sepsis and meningitis. In: Remington JS, Klein JO, editors. Infectious Disease of the fetus and newborn infants. Philadelphia: W. B. Saunders; 2001. p. 943-98.

20. Oğuz SS, Kızılelma A, Erdeve O, Zergeroğlu S, Saygan S, Kılıç S, et al. A case of neonatal meningitis due to Listeria monocytogenes serotype 1/2b. Mikrobiyol Bul. 2011;45 (3):541-5.

21. Farber JM, Peterkin PI, Carter AO, Varughese PV, Ashton FE, Ewan EP. Neonatal listeriosis due to cross-infection confirmed by isoenzyme typing and DNA fingerprinting. J Infect Dis. 1991;163(4):927-8.

22. Softić I, Tahirović H, Skokić F, Tihić N, Di Ciommo V, Auriti C. An outbreak of nosocomial infection with Acinetobacter baumanni in the neonatal intensive care unit of the Department of paediatrics, University clinical centre Tuzla, Bosnia and Herzegovina. Paediatrics Today. 2013:9(2):163-69.

23. Wankaew N, Jirapradittha J, Kiatchoosakun P. Neonatal morbidity and mortality for repeated cesarean section vs. normal vaginal delivery to uncomplicated term pregnancies at Srinagarind Hospital. J Med Assoc Thai. 2013;96(6):654-60.

24. Zanella P, Bogana G, Ciullo R, Zambon A, Serena A, Albertin MA. Chorioamnionitis in the delivery room. Minerva Pediatr. 2010;62(3):151-3. 